Revista Colombiana de Obstetricia y Ginecología Vol. 63 No. 2 • Abril-Junio 2012 • (155-162)

RePORTE DE CASO

\title{
EXIT: TRATAMIENTO EXÚTERO INTRAPARTO. REPORTE DE CASO Y REVISIÓN DE LA LITERATURA
}

\section{EXIT: ex-uitero intrapartum treatment. A case report and literature review}

Juan Camilo Ospina-García, M.D. ${ }^{\text {, }}$ Carolina Wuesthoff-Prieto, M.D. ${ }^{2}$, Santiago Eslava-Cerón, M.D. ${ }^{3}$

Recibido: enero 19/12 - Aceptado: junio 22/12

\section{RESUMEN}

Introducción y objetivo: la técnica Tratamiento exútero intraparto (EXIT) permite el aseguramiento de la vía aérea fetal mientras se mantiene el soporte uteroplacentario íntegro. El objetivo de reportar este caso es describir el procedimiento y hacer una revisión de la literatura mundial respecto a consideraciones anestésicas y complicaciones asociadas.

Materiales y métodos: presentamos el caso de una neonato femenina de 39 semanas de gestación que nace mediante la técnica Tratamiento exútero intraparto (EXIT) debido a una potencial obstrucción de la vía aérea detectada en una ecografía de tercer trimestre. Se realizó una búsqueda sistemática usando las palabras clave: Tratamiento exútero intraparto (EXIT), masas cervicales congénitas y CHAOS en las bases de datos Medline via Pub Med, Ovid, y la base latinoamericana SciELO.

1 Otorrinolaringólogo pediatra. Profesor Asistente. Jefe Unidad de Otorrinolaringología, Hospital Universitario San Ignacio, Pontificia Universidad Javeriana. Bogotá, Colombia. juan.ospina@gmail.com

2 Residente III de Otorrinolaringología, Pontificia Universidad Javeriana, Hospital Universitario San Ignacio. Bogotá, Colombia.

3 Anestesiólogo, Clínica del Country. Bogotá, Colombia.
Resultados: se encontraron un total de 183 artículos, de los cuales 76 estaban relacionados directamente con el tema, de estos se seleccionaron 8 reportes de caso, 2 series de casos y 14 revisiones del tema.

Conclusiones: la estrategia EXIT prueba ser una herramienta útil en el adecuado manejo de los neonatos con obstrucciones congénitas de la vía aérea. Palabras clave: tratamiento exútero intraparto (EXIT), masas cervicales congénitas, síndrome de obstrucción congénita de la vía aérea superior (CHAOS).

\section{ABSTRACT}

Introduction and objective: The EXIT procedure (ex-utero intrapartum treatment) is aimed at securing the fetal high airway whilst maintaining integral uteroplacentary circulation. The purpose of reporting this case was to describe the procedure and review the worldwide literature regarding anesthetic considerations and associated complications.

Materials and methods: The case of a 39-week gestation female neonate is presented; she was born at the Clínica del Country (a private highlevel healthcare general hospital located in Bogota, 
Colombia) by the ex-utero intrapartum treatment (EXIT) technique due to a potential obstruction of the airway detected during third-trimester echography. A systematic search was made of Medline databases via PubMed, Ovid, and the SciELO Latin-American database using the following key words: EXIT, ex-utero intrapartum treatment, congenital cervical mass, CHAOS.

Results: 183 articles were found, of which 76 were directly related to the topic; 8 case reports, 2 case series and 14 reviews of the topic were selected.

Conclusion: The EXIT strategy has proved to be a useful tool in the suitable management of neonates suffering congenital obstruction of the airway.

Key words: Ex-utero intrapartum treatment (EXIT), congenital cervical mass, congenital high airway obstruction syndrome (CHAOS).

\section{INTRODUCCIÓN}

La detección prenatal de malformaciones congénitas cervicales, craneofaciales e intratorácicas se ha optimizado ampliamente en los últimos años permitiendo su diagnóstico precoz gracias a las diferentes herramientas con las que se cuenta en la actualidad, tales como el ultrasonido de alta definición, el ultrasonido con reconstrucción 3D, o la resonancia magnética nuclear fetal. En estos casos, el aseguramiento de la vía aérea mediante una estrategia intraparto es de vital importancia para disminuir posibles complicaciones $(1,2)$.

La estrategia EXIT, sigla anglosajona que traducida al español significa Tratamiento exútero intraparto, surge como modificación a la técnica Operating On Placental Support (OOPS) (3), y se utilizó inicialmente en la década de los ochenta para retirar clips traqueales que habían sido colocados in útero en fetos con casos severos de hernia diafragmática congénita (4); luego en 1997 Mychaliska et al. acuñan el acrónimo y estandarizan el procedimiento estableciendo las guías de manejo, en donde se resalta la importancia del manejo multidisciplinario entre los servicios de Ginecoobstetricia, Anestesiología, Neonatología,
Radiología y Otorrinolaringología. Con esta técnica se busca asegurar la vía aérea fetal antes de interrumpir el soporte de oxígeno proveniente de la circulación útero-placentaria (5).

El objetivo de este reporte de caso es describir las condiciones que se requieren para realizar el procedimiento, describir los pasos que incluye, las consideraciones de la fisiología materna y fetal que se deben tener en cuenta, y hacer una revisión la literatura mundial respecto a consideraciones anestésicas y complicaciones asociadas. Hasta donde sabemos, se trata del segundo caso reportado en la literatura biomédica colombiana (6).

\section{REPORTE DE CASO}

Presentamos el caso de una mujer de 34 años, paciente de la Clínica del Country en Bogotá, Colombia. Esta es una institución privada de atención generaI y alta complejidad que cuenta con una unidad materno-fetal de alto nivel y atiende paciente del régimen de aseguramiento privado y contributivo. La paciente ingresa con un embarazo al término de 39 semanas remitida por presentar una potencial obstrucción aguda de la vía aérea durante el parto, debido a una masa que en apariencia ocupaba la totalidad de la cavidad oral, evidenciada mediante ecografía 3D (figura 1). Se decide recibir al neonato mediante la estrategia EXIT, con la que se obtiene un buen resultado perinatal y materno. Al valorar a la recién nacida durante su adaptación se encontró que la masa no obstruía la vía aérea de una manera importante, y se podía rechazar por lo que fue posible su extubación poco después del nacimiento (figura 2). El diagnóstico final determinó que se trataba de un teratoma con origen en la base del cráneo, que descendía a través de la cavidad nasal y el paladar hendido hasta terminar alojado en la cavidad oral (figura 3). La masa fue intervenida y resecada de forma exitosa por el neurocirujano pocos días después.

Se solicitó autorización a la familia para la publicación del caso y se tomaron todas las precauciones para proteger la identidad de la paciente del estudio. 


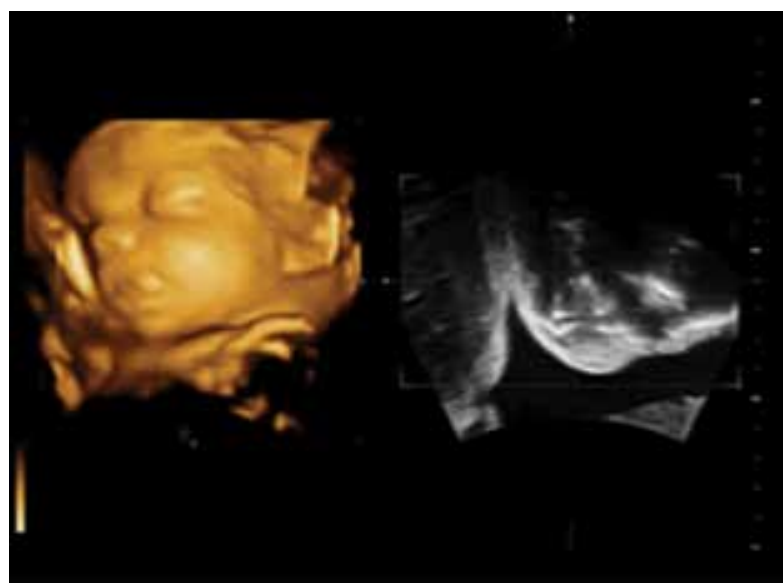

Figura 1. Ecografía de tercer trimestre de la paciente en donde se muestra la masa en cavidad oral con riesgo de obstruir la vía aérea en el nacimiento.

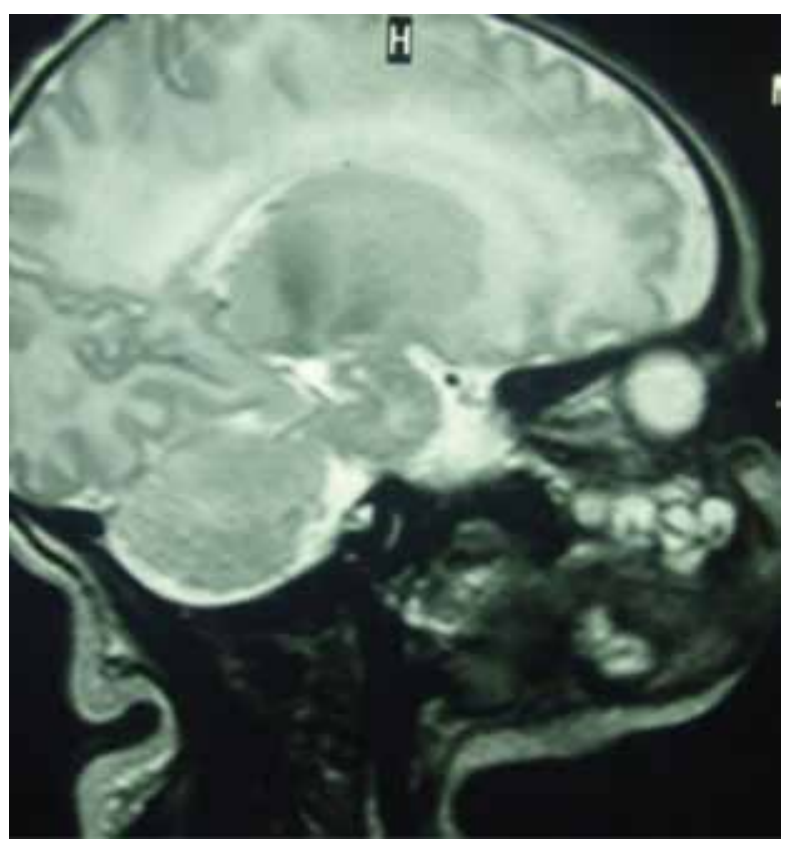

Figura 3. RMN de la paciente que muestra el origen de la masa en la base del cráneo.

\section{MATERIALES Y MÉTODOS}

Se revisaron las bases de datos Medline, Ovid, y la base latinoamericana SciELO en el periodo comprendido entre los años 1995 y 2012. Se utilizaron las siguientes palabras clave: Tratamiento exútero intraparto (EXIT), masas cervicales congénitas y CHAOS.

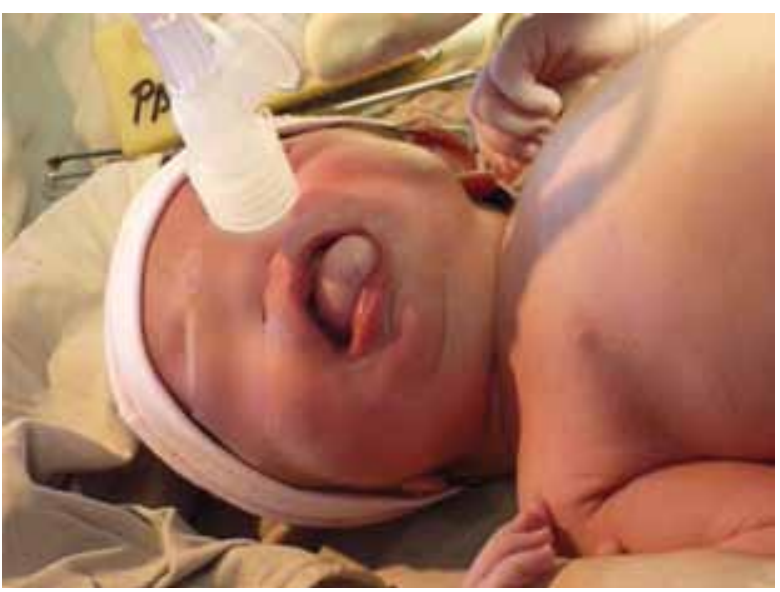

Figura 2. Paciente adecuadamente adaptada y recibida mediante técnica EXIT, quien tolera la extubación.

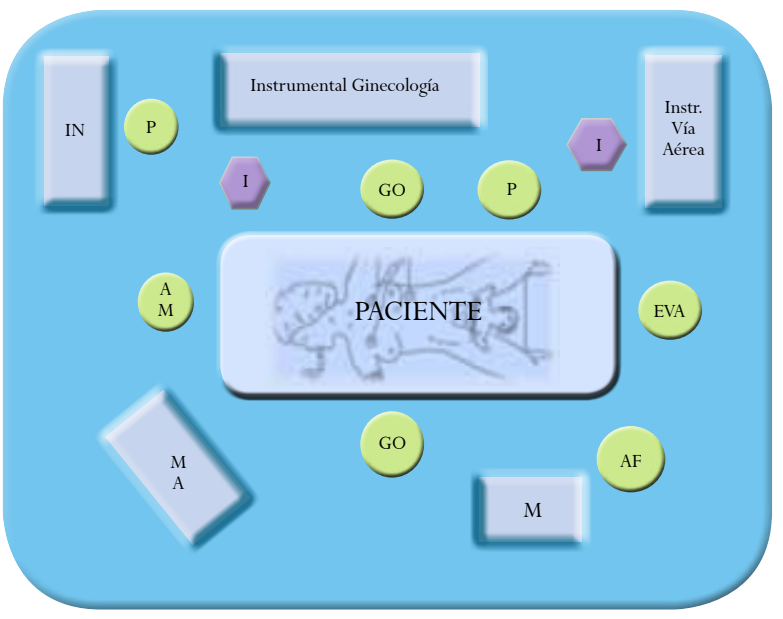

Figura 4. Diagrama que indica la posición de cada uno de los integrantes del equipo EXIT dentro de la sala de cirugía para optimizar el espacio.

IN: incubadora. P: pediatra neonatólogo. I: instrumentadora. GO: ginecoobstetra. AF: anestesiólogo feto. AM: anestesiólogo madre. MA: máquina de anestesia. M: monitor fetal. EVA: experto en vía aérea.

\section{RESULTADOS}

Se seleccionaron 183 artículos, de los cuales 76 estaban relacionados directamente con el tema, de estos se escogieron 8 reportes de caso, 2 series de casos y 14 revisiones del tema, para un total de 24 artículos. 


\section{Descripción del procedimiento EXIT}

Condiciones que se requieren para realizar el procedimiento: idealmente deben estar presentes en el quirófano dos anestesiólogos (uno para la madre y uno para el bebé), un ecografista, dos obstetras, un otorrinolaringólogo experto en vía aérea pediátrica y un neonatólogo, además del soporte de enfermería e instrumentación. Las personas se deben ubicar en posiciones estratégicas que permitan su desenvolvimiento fácil y efectivo durante el procedimiento (figura 4). Las enfermeras que asisten en la sala deben tener preparación o entrenamiento previo en la técnica EXIT, y se dividen en dos grupos: uno para asistir lo relacionado con la madre y otro para asistir al feto $(7,8)$.

La madre se posiciona en decúbito supino con ligera inclinación hacia el decúbito lateral izquierdo para evitar la compresión aortocava, mejorar el retorno venoso y asegurar la presión de perfusión uterina, evitando la hipoxia fetal. Con el mismo fin se utiliza un sistema de compresión neumática intermitente en los miembros inferiores $(9,10)$.

El instrumental de vía aérea fetal está preparado antes del inicio de la cesárea e incluye tubos endotraqueales de diámetros externos diferentes, ambú, cánula de Gedel, broncoscopios y lentes rígidos, succiones rígidas, laringoscopios, laringoscopio de fibra óptica flexible, y el instrumental y las cánulas para traqueostomía (7) (figura 5).

\section{Pasos del procedimiento}

\section{Anestesia}

Se realiza una secuencia rápida para la intubación. La inducción anestésica se efectúa con remifentanil, rocuronio y propofol. El segundo paso anestésico y la hipotonía uterina ideal se consiguen con sevofluorano a 2 y 3 CAM. Se mantiene infusión continua de remifentanil para mantenimiento del plano materno-fetal, hipotensión y tocolisis. Este medicamento es ideal para este tipo de procedimientos puesto que cruza efectivamente la barrera útero-placentaria y produce inmovilización fetal efectiva, y como el neonato será intubado al final del

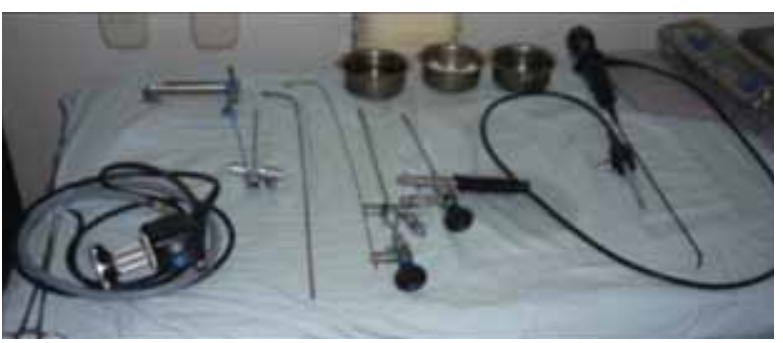

Figura 5. Instrumental que debe estar disponible para el otorrinolaringólogo experto de vía aérea antes del procedimiento.

procedimiento, la depresión respiratoria que produce no es motivo de preocupación $(7,11,12,13)$.

En el caso de nuestra paciente fue de $0,10 \mathrm{mcg} /$ $\mathrm{kg} /$ min debido a que la concentración de halogenado utilizada compensó estos efectos. Para mantener la presión de perfusión placentaria se utilizó efedrina titulada cuidando que la presión sistólica de la madre no fuera menor a $100 \mathrm{mmHg}(2,14)$.

\section{Cesárea y extracción parcial del feto}

Posterior a la anestesia se realiza el mapeo ecográfico de la ubicación placentaria de manera que esta no sea lesionada durante la histerotomía, al comprobar que la placenta está en posición habitual se realiza la incisión de Pfannenstiel. Se utiliza un sistema especial de grapas absorbibles para evitar la hemorragia secundaria a la relajación sostenida del útero durante el procedimiento (15).

A continuación se expone la cabeza fetal, la cual es orientada de manera que sea accesible para valorar la vía aérea e intentar la intubación. Se expone también, por protocolo, el miembro superior derecho del neonato para efectos de monitorización, o en caso de requerirse una línea endovenosa independiente para administración de medicamentos (figura 6). Varias partes fetales pueden ser expuestas dependiendo de la indicación para esta cirugía. La exposición fetal debe ser lo más discreta posible para evitar la pérdida de calor, el compromiso del cordón o el desprendimiento prematuro de la placenta, así como la pérdida de volumen uterino que estimularía las contracciones 
Figura 6. Pasos del procedimiento EXIT

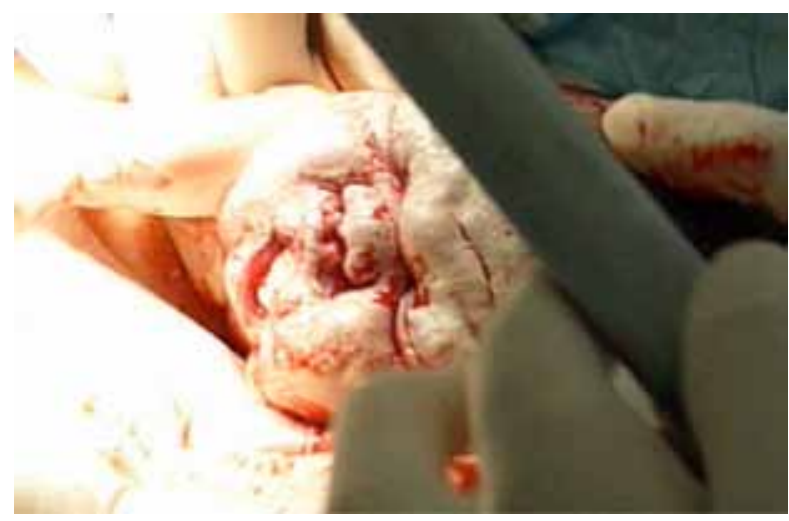

A: exposición de cabeza fetal.

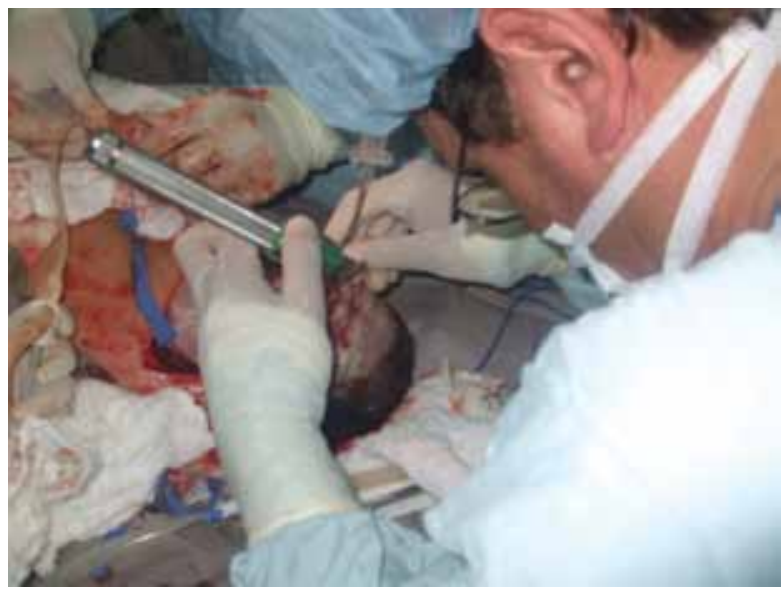

Figura 7. Intento de intubación con feto todavía parcialmente in utero.

$(11,16)$. Algunos autores plantean el uso de irrigación con lactato de Ringer tibio dentro de la cavidad uterina como método de compensación de pérdida de volumen a la vez que mantiene caliente al feto (10). En el caso presentado se expuso la cabeza y el miembro superior derecho. No se utilizó irrigación con lactato de Ringer.

\section{Aseguramiento de la vía aérea y extracción del feto}

El protocolo EXIT incluye: laringoscopia-broncoscopia o nasofibrolaringoscopia para asegurar de manera adecuada la vía aérea, realizar una evaluación más detallada del feto, y determinar si existen otras malformaciones o alteraciones funcionales de la vía aérea asociadas. En muchos casos la vía

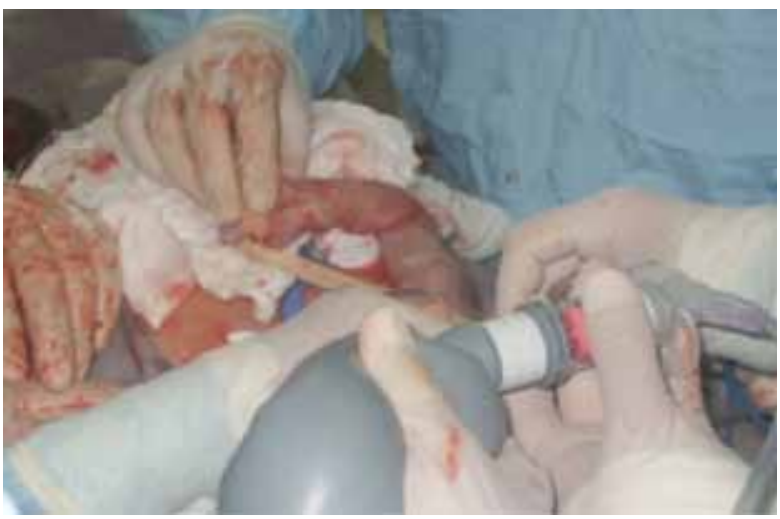

B: exposición de miembro superior derecho fetal.

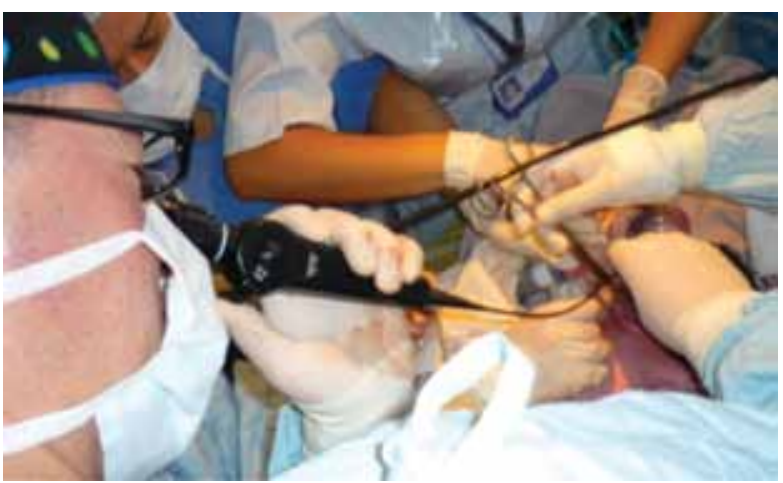

Figura 8. Valoración de la vía aérea neonatal inmediatamente después de su extracción completa.

aérea está distorsionada, por tanto, se requerirá la ayuda de una broncoscopia rígida, usualmente con un broncoscopio 2,5 o 3,0 para definir la anatomía de la misma y guiar el tubo endotraqueal armado sobre el broncoscopio. Cuando el paso del broncoscopio rígido es bloqueado, se utilizaría el flexible, el cual también es útil para confirmar la posición del tubo. Por medio del broncoscopio también es posible la administración de surfactante pulmonar. Finalmente, la última opción es la realización de la traqueostomía, tanto casos en los que la colocación del tubo no ha sido posible como cuando se prevé una intubación orotraqueal (IOT) prolongada. Es importante tener en cuenta que en casos de masas cervicales la traqueostomía puede localizarse más alta, ya que la tráquea tiende a ser desplazada hacia anterior y arriba $(4,5,6,15,17)$. 
En nuestro caso no fue necesario realizar nasofibrolaringoscopia ya que se realizó laringoscopia directa con hojas 0 o 00 de Miller e intubación exitosa con TET 3.0 (figura 7). Al comprobarse el control de la vía aérea se extrajo el feto pinzando el cordón umbilical para posteriormente realizar una evaluación completa del feto (figura 8).

\section{Reversión de la tocolisis, el paso final}

Una vez pinzado el cordón, la relajación uterina se convierte en un factor de sangrado por lo que debe revertirse disminuyendo la concentración del halogenado rápidamente y administrando oxitocina y metilergonovina $(1,10,12,13,15,17,18)$. En la paciente que presentamos se usó carbetocina justo antes de extraer la placenta.

\section{DISCUSIÓN}

A pesar de no ser una práctica común en Latinoamérica, en países como Estados Unidos la estrategia EXIT es utilizada con frecuencia. Se encuentran reportes de series de casos, como la de 43 pacientes manejados en el Children's Hospital de Philadelphia entre 1996 y 2002, en los cuales la indicación más frecuente fue la presencia de masas cervicales y el síndrome de obstrucción congénita de la vía aérea (CHAOS) (11). Sin embargo, existen muchas otras indicaciones como el manejo de atresia laringotraqueal, micrognatia severa, masas torácicas, mediastinales y pericárdicas, oxigenación extracorpórea transmembranosa, e incluso separación de gemelos unidos, entre otras $(1,8,9,15,16,19)$. La técnica quirúrgica en el EXIT depende de varios factores como localización de la placenta, edad gestacional y, obviamente, la naturaleza de la anomalía fetal que indica la realización del procedimiento $(4,7,20)$. Su duración es variable, el máximo periodo de tiempo considerado seguro tanto para la madre como para el feto es de 60 min (4). En casos en los que se ha utilizado bypass útero-placentario se ha logrado prolongar de 2,5 hasta 3 horas $(1,3)$.

\section{Consideraciones anestésicas especiales}

Es muy importante anotar que los riesgos anestésicos se aumentan, no solo por las características anestésicas especiales de esta técnica, sino debido también a los cambios fisiológicos normales del embarazo.

La anestesia durante la estrategia EXIT se planea para: el parto, el mantenimiento sostenido de la tocolisis, el control de la tensión arterial materna, la posterior recuperación del tono uterino, y para asegurar la anestesia y la vía aérea fetal. A diferencia de una cirugía convencional, no se evita la exposición del útero y el feto a los agentes anestésicos inhalados $(7,8,9,12)$.

Las condiciones más importantes para tener en cuenta son: el mantenimiento de la "hipotonía uterina controlada" para mantener la circulación útero-placentaria, la conservación al máximo del volumen uterino para evitar el desprendimiento placentario, el sostenimiento de un plano anestésico profundo materno pero con tensión arterial en niveles apropiados, y el mantenimiento de un buen nivel de anestesia fetal sin llegar a causar hipoxia o depresión cardíaca $(1,3,7,12,13)$.

La inducción es rápida, generalmente involucra el uso de remifentanil y el mantenimiento se realiza con anestésico inhalado; el halotano y el isofluorano son los utilizados en cesáreas; por lo general, como se describió antes, se prefiere el uso de sevofluorano por su rápida titulación y reversibilidad $(6,9,19)$. Se puede administrar concomitantemente tiopental, succinilcolina, fentanil o remifentanil en infusión para mantener el plano, la tocolisis y la hipotensión $(7,11,12,13)$.

El efecto tocolítico del anestésico inhalado puede ser potenciado por medicamentos como el sulfato de magnesio, los beta-adrenérgicos, los AINES, la nitroglicerina, la terbutalina o la indometacina administrada en el preoperatorio, aunque esta última no es usada con frecuencia debido a sus efectos sobre las plaquetas, el ductus arterioso neonatal y la función renal $(3,6,9)$. 
El equilibrio entre el mantenimiento de la tensión arterial materna por encima del límite de lo nocivo mientras se conserva una adecuada vasodilatación se realiza mediante una infusión de efedrina $(2,4,5,8$, $9,13)$; otros fármacos usados para ese fin incluyen la nitroglicerina, la fenilefrina titulada y la angiotensina II, pero la efedrina es más popular por tener efectos más selectivos con el órgano blanco $(2,7,11,20)$. Se debe lograr un adecuado plano anestésico en el feto independientemente de si la anestesia materna es epidural, la aplicación de los anestésicos se realiza de forma intramuscular. Sin embargo, si la anestesia materna es general, puede ser suficiente, especialmente si se utiliza remifentanil (12). El movimiento o la taquicardia fetal indican un inadecuado plano anestésico $(1,3,9)$. Esto dificulta la laringoscopia y disminuye el tiempo disponible de circulación fetoplacentaria (1).

La idea es que el tiempo entre la inducción y el pinzamiento del cordón sea el menor posible, de manera que la concentración de anestésico inhalado sea poca a fin de evitar la hemorragia $(8,9,12,20)$.

Al terminar el procedimiento la relajación uterina, vital para la realización del EXIT, puede convertirse en una amenaza para la madre si no se revierte adecuada y rápidamente en el momento preciso; para este fin es necesario disminuir el volumen alveolar del anestésico inhalado rápidamente antes del pinzamiento del cordón; posteriormente se administra oxitocina intravenosa, la cual se continúa en goteo durante el posoperatorio $(1,3,17,18,13,19)$.

Por último, dentro del protocolo EXIT no hay lugar para permitir la respiración espontánea, se inicia con una laringoscopia directa y, de ser posible, se procede a intubar $(4,21-23)$.

\section{Complicaciones inherentes al procedimiento}

Las complicaciones relacionadas con el procedimiento o la técnica EXIT incluyen: la pérdida sanguínea materna, que varía en promedio entre
500 y 800 cc, así como la bradicardia, encefalopatía hipóxico-isquémica, hemorragia, acidosis por exposición prolongada a anestésicos inhalados y muerte fetal $(1,7,9)$.

\section{CONCLUSIONES}

La estrategia EXIT surge como una herramienta útil en el adecuado manejo de los neonatos con obstrucciones congénitas de la vía aérea. Se requiere el trabajo en equipo de un grupo multidisciplinario para obtener los mejores resultados. Se deben tener en cuenta los cambios fisiológicos de la mujer embarazada, una adecuada relajación uterina por un tiempo limitado, y garantizar un rápido acceso a la vía aérea del feto.

\section{AGRADECIMIENTOS}

A todo el personal de la Clínica del Country, Bogotá, Colombia, que participó en la atención de la madre y la bebé presentadas en este artículo.

\section{REFERENCIAS}

1. Morris LM, Lim F-Y, Crombleholme TM et al. Ex Utero Intrapartum Treatment Procedure: A Peripartum Management Strategy In Particularly Challenging Cases. J Pediatr 2009;154:126-31.e3.

2. Chiu H-H, Hsu W-C, Shih J-C, Tsao P-N, Hsieh W-S, Chou H-C. The Exit (Ex Utero Intrapartum Treatment) Procedure. J Formos Med Assoc 2008;107:745-8.

3. Skarsgard ED, Chitkara U, Krane EJ, Riley ET, Halamek LP, Dedo HH. The OOPS Procedure (Operation on Placental Support): In utero airway management of the fetus with prenatally diagnosed tracheal obstruction. J Pediatr Surg 1996;31:826-8.

4. MacKenzie TC, Crombleholme TM, Flake AW. The ex-utero intrapartum treatment. Curr Opin Pediatr 2002;14:453-8.

5. Mychaliska GB, Bealer JF, Graf JL, Rosen MA, Adzick NS, Harrison MR. Operating on placental support: the ex utero intrapartum treatment procedure. J Pediatr Surg 1997;32:227-30.

6. Ferrer L, Paredes P. Manejo anestésico de paciente obstétrica sometida a EXIT. Rev Colomb Anestesiol 2008;36:297-9. 
7. Liechty KW. Ex-utero intrapartum therapy. Semin Fetal Neonatal Med. 2010;15:34-9.

8. Hirose S, Harrison MR. The ex utero intrapartum treatment (EXIT) procedure. Semin Neonatol 2003;8:207-14.

9. Marwan A, Crombleholme TM. The EXIT procedure: principles, pitfalls, and progress. Semin Pediatr Surg 2006;15:107-15.

10. Fink RJ, Allen TK, Habib AS. Remifentanil for fetal immobilization and analgesia during the ex utero intrapartum treatment procedure under combined spinal-epidural anaesthesia. $\mathrm{Br} \mathrm{J}$ Anaesth 2011;106:851-5.

11. Kudo T, Kimura F, Hashimoto H, Wada M, Hirota K. Anesthetic management for EXIT (ex-utero intrapartum treatment) of a twin gestation: one normal and one with a large epignathus. Masui 2012;61:307-10.

12. Lee H, Ryu JW, Kim DY, Lee GY. Anesthetic management of the ex utero intrapartum treatment (EXIT) procedure. Korean J Anesthesiol 2010;59:S154-S7.

13. Schwartz DA, Moriarty KP, Tashjian DB, Wool RS, Parker RK, Markenson GR, et al. Anesthetic Management of the EXIT (Ex Utero Intrapartum Treatment) Procedure. J Clin Anesth 2001;13:387-91.

14. Ioscovich A, Shen O, Sichel J-Y, Lajos Y, Orkin $\mathrm{D}$, Bromiker R, et al. Remifentanil-nitroglycerin combination as an anesthetic support for ex utero intrapartum treatment (EXIT) procedure. J Clin Anesth 2011;23:142-4.

15. Olutoye OO, Olutoye O. EXIT procedure for fetal neck masses. Curr Opin Pediatr 2012;24:386-93.
16. Walker P, Cassey J, O'Callaghan S. Management of antenatally detected fetal airway obstruction. Int J Pediatr Otorhinolaryngol 2005;69:805-9.

17. Laje P, Johnson MP, Howell LJ, Bebbington MW, Hedrick HL, Flake AW, et al. Ex utero intrapartum treatment in the management of giant cervical teratomas. Journal of Pediatric Surgery 2012;47:1208-16.

18. Garcia P, Olutoye OO, Ivey RT, Olutoye OA. Case Scenario: Anesthesia for Maternal-Fetal Surgery. The Ex Utero Intrapartum Therapy (EXIT) Procedure. Anesthesiology 2011;114:1446-52.

19. Woodard TD, Yong S, Hotaling AJ. The Ex Utero Intrapartum Treatment (EXIT) procedure used for airway control in a newborn with cervical fetus in fetu: A rare case. Int J Pediatr Otorhinolaryngol 2006;70:1989-94.

20. Zadra N, Giusti F, Midrio P. Ex utero intrapartum surgery (EXIT): indications and anaesthetic management. Best Pract Res Clin Anaesthesiol 2004;18:259-71.

21. Cauldwell CB. Anesthesia for fetal surgery. Anesthesiol Clin North America. 2002;20:211-26.

22. Lazar DA, Olutoye OO, Moise KJ, Ivey RJ, Johnson A, Ayres N, et al. Ex-utero intrapartum treatment procedure for giant neck masses-fetal and maternal outcomes. J Pediatr Surg 2011;46:817-22.

23. Choleva AJ. Anesthetic management of a patient undergoing an ex utero intrapartum treatment (EXIT) procedure: a case report. AANA J 2011;79:497-503. 